\title{
TAUTOLOGICAL CLASSES OF THE STACK OF RATIONAL NODAL CURVES
}

\author{
DAMIANO FULGHESU
}

\begin{abstract}
This is the second in a series of three papers in which we investigate the rational Chow ring of the stack $\mathfrak{M}_{0}$ consisting of nodal curves of genus 0 . Here we define the basic classes: the classes of strata and the Mumford classes.
\end{abstract}

\section{INTRODUCTION}

In the first paper Fulg we described the stack $\mathfrak{M}_{0}$, its stratification by nodes and more precisely by topological types given by dual graphs. When the graph $\Gamma$ corresponding to a curve $C$ has maximal multiplicity 3 , we have an equivalence:

$$
\mathfrak{M}_{0}^{\Gamma} \equiv \mathfrak{M}_{0}^{\Gamma} \simeq \operatorname{BAut}(C) .
$$

This explicit description allows us to describe completely two kinds of classes.

Strata classes: for each tree $\Gamma$ with $\delta$ edges and maximal multiplicity 3 , we get a class $\gamma_{\Gamma}$ of codimension $\delta$ in $\mathfrak{M}_{0}$ : the class of the closure of $\mathfrak{M}_{0}^{\Gamma}$ in $\mathfrak{M}_{0}$. We also compute the restriction of each $\gamma_{\Gamma}$ to all the rings $A^{*}\left(\mathfrak{M}_{0}^{\Gamma^{\prime}}\right) \otimes \mathbb{Q}$ for any other tree $\Gamma^{\prime}$ with maximal multiplicity 3 (Proposition 3.14).

Mumford classes: as the map from the universal curve is not locally projective we cannot define these classes as in the usual sense because in Kresch's theory one has only pushforwards along projective morphisms.

However we are able to extend these classes in the following way (Section 4 ): let $\mathcal{C} \stackrel{\Pi}{\rightarrow} \mathfrak{M}_{0}$ be the universal curve. Call $K \in A^{1}(\mathcal{C}) \otimes \mathbb{Q}$ the first Chern class of the relative dualizing sheaf $\omega_{\mathcal{C} / \mathfrak{M}_{0}}$, and set

$$
\kappa_{i}=\Pi_{*}\left(K^{i+1}\right) .
$$

Here we encounter a hard technical problem: the morphism $C \rightarrow \mathfrak{M}_{0}$ is not projective (not even represented by schemes, as we have seen in Fulg ). 
If $n \leq 3$ then the pushforward $\Pi_{*} \omega_{\mathcal{C} / \mathfrak{M}_{0}}^{\vee}$ is a locally free sheaf of rank 3 on the the open substack $\mathfrak{M}_{0}^{\leq 3}$ (this fails for curves with 4 nodes). Hence we have Chern classes $c_{1}, c_{2}$ and $c_{3}$ of $\Pi_{*} \omega_{\mathcal{C}}^{\vee} \mathfrak{M}_{0}$ in $A^{*}\left(\mathfrak{M}_{0}^{\leq 3}\right)$.

We have a pushforward $\Pi_{*} A^{*}\left(\mathrm{C}^{\Gamma}\right) \otimes \mathbb{Q} \rightarrow A^{*}\left(\mathfrak{M}_{0}^{\Gamma}\right) \otimes \mathbb{Q}$ along the restriction $\mathcal{C}^{\Gamma} \stackrel{\Pi}{\rightarrow} \mathfrak{M}_{0}^{\Gamma}$ of the universal curve, because the stacks involved are quotient stacks, and arbitrary proper pushforwards exists in the theory of Edidin and Graham. This allows, with GrothendieckRiemann-Roch, to compute the restriction of the Mumford classes to each $A^{*}\left(\mathfrak{M}_{0}^{\Gamma}\right) \otimes \mathbb{Q}$ (Proposition 4.3 ), even without knowing that the Mumford classes exist. It turns out that for each tree $\Gamma$ with at most 3 nodes, the Mumford classes in $A^{*}\left(\mathfrak{M}_{0}^{\Gamma}\right) \otimes \mathbb{Q}$ are polynomials in the restrictions of $\mathcal{c}_{1}, c_{2}$ and $\mathcal{c}_{3}$, thought of as elementary symmetric polynomials in three variables. Then we define the classes $\kappa_{i} \in A^{*}\left(\mathfrak{M}_{0}^{\leq 3}\right) \otimes \mathbb{Q}$ as the suitable polynomials in the $\mathfrak{c}_{1}, \boldsymbol{c}_{2}$ and $\mathfrak{c}_{3}$ (Definition 4.4).

Since $A^{*}\left(\mathfrak{M}_{0}^{\leq 3}\right) \otimes \mathbb{Q}$ injects into the product of $A^{*}\left(\mathfrak{M}_{0}^{\Gamma}\right) \otimes \mathbb{Q}$ over trees with at most three nodes (Proposition 2.11), this gives the right definition.

\section{Chow Rings of StRata}

In this paper we work on a fixed tree $\Gamma$ with maximal multiplicity 3 and $\delta$ edges. We indicate with $\Delta_{1}, \Delta_{2}, \Delta_{3}$ the sets of vertices that belongs respectively to one, two and three edges (and with $\delta_{1}, \delta_{2}, \delta_{3}$ their cardinalities).

We restrict the universal curve $\mathcal{C} \rightarrow \mathfrak{M}_{0}$ to $\mathfrak{M}_{0}^{\Gamma}$

$$
C^{\Gamma} \stackrel{\Pi}{\rightarrow} \mathfrak{M}_{0}^{\Gamma}
$$

and consider the normalization (see [Vis] Definition 1.18)

$$
\widehat{C}^{\Gamma} \stackrel{N}{\rightarrow} C^{\Gamma} \text {. }
$$

Remark 2.1. Given a curve $C \stackrel{\pi}{\rightarrow} T$ in $\mathfrak{M}_{0}^{\Gamma}$ (that is to say a morphism $T \stackrel{f}{\rightarrow} \mathfrak{M}_{0}^{\Gamma}$ ) we define $\widehat{C}$ as $T \times_{\mathfrak{M}_{0}^{\Gamma}} \widehat{C}^{\Gamma}$. We have the following cartesian diagram

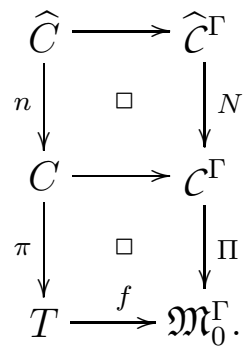


We notice that when $T$ is a reduced and irreducible scheme $\widehat{C} \stackrel{n}{\rightarrow} C$ is the normalization.

The map $\pi n: \widehat{C} \rightarrow T$ is proper as $\Pi N: \mathcal{C}^{\Gamma} \rightarrow \mathfrak{M}_{0}^{\Gamma}$ is. Then there exists a finite covering $\widetilde{T} \rightarrow T$ (see [Knu Chapter 5, Theorem 4.1) such that we have the following commutative diagram

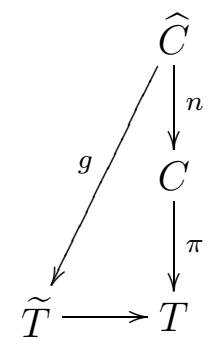

where the map $g$ has connected fibers.

Definition 2.2. We define $\widetilde{\mathfrak{M}}_{0}^{\Gamma}$ as the fibered category on $\mathcal{S} \mathfrak{h}_{\mathbb{C}}$ whose objects are rational nodal curves $C \stackrel{\pi}{\rightarrow} T$ in $\mathfrak{M}_{0}^{\Gamma}$ equipped with an isomorphism $\varphi: \coprod^{\Gamma} T \rightarrow \widetilde{T}$ over $T$ and maps are morphisms in $\mathfrak{M}_{0}^{\Gamma}$ that preserve isomorphisms.

Straightforward arguments show the following

Lemma 2.3. The forgetful morphism

$$
\widetilde{\mathfrak{M}}_{0}^{\Gamma} \rightarrow \mathfrak{M}_{0}^{\Gamma}
$$

is representable finite étale and surjective.

We have a more explicit description of $\widetilde{\mathfrak{M}_{0}^{\Gamma}}$ : if we call $\mathcal{M}_{0, i}^{n}$ the stack of rational curves with $n$ nodes and $i$ sections, we can exhibit an equivalence

$$
\widetilde{\mathfrak{M}}_{0}^{\Gamma} \cong \prod_{\alpha \in \Gamma}\left(\mathcal{M}_{0, e(\alpha)}^{0}\right)
$$

where, for each $\alpha \in \Gamma, e(\alpha)$. The proof of this is straightforward, however in the following we assume that the maximal multiplicity $k$ of $\Gamma$ is at most 3 . In this case, as we have seen in Fulg:

$$
\mathfrak{M}_{0}^{\Gamma}=\operatorname{BAut}(C)
$$

where $\operatorname{Aut}(C)$ is the group $\operatorname{Aut}(\Gamma) \ltimes\left(E^{\Delta_{1}} \times \mathbb{G}_{\mathbf{m}}^{\Delta_{2}}\right)$. Let us call $\sigma$ the order of Aut $\Gamma$.

The étale covering of degree $\sigma$

$$
\widetilde{\mathfrak{M}}_{0}^{\Gamma} \stackrel{\phi}{\rightarrow} \mathfrak{M}_{0}^{\Gamma}
$$

becomes

$$
\mathrm{BH} \stackrel{\phi}{\rightarrow} \operatorname{BAut}(C)
$$


where $H$ is the group $E^{\Delta_{1}} \times \mathbb{G}_{\mathbf{m}}^{\Delta_{2}}$.

In order to compute the Chow ring on each stratum we only need equivariant intersection theory.

Let $\Gamma$ be a tree of maximal multiplicity at most 3 and let $C$ be the unique isomorphism class of curves of topological type $\Gamma$. Let us fix coordinates $[X, Y]$ on each component of $C$ such that

- on components with one node the point $[1,0]$ is the node,

- on components with two nodes the points $[0,1]$ and $[1,0]$ are the nodes,

- on components with three nodes the points $[0,1],[1,1]$ and $[1,0]$ are the nodes.

Let us define on each component

$$
0:=[0,1] \quad 1:=[1,1] \quad \infty:=[1,0]
$$

Proposition 2.4. Given $\Gamma$ as above, the Chow ring $A^{*}\left(\mathfrak{M}_{0}^{\Gamma}\right)$ is

$$
A_{A u t(C)}^{*} \otimes \mathbb{Q} \cong\left(\mathbb{Q}\left[t_{\Delta_{1}}, r_{\Delta_{2}}\right]\right)^{A u t(\Gamma)}
$$

where the action of an element $g \in \operatorname{Aut}(\Gamma)$ on $\mathbb{Q}\left[x_{\Delta_{1}}, y_{\Delta_{2}}\right]$ is the obvious permutation on the $\Delta_{1} \cup \Delta_{2}$ variables together with multiplication by (-1) of r-variables corresponding to components of which $g$ exchanges 0 and $\infty$.

Proof. The group $E^{\Delta_{1}} \times \mathbb{G}_{\mathbf{m}}^{\Delta_{2}}$ is a normal subgroup of $\operatorname{Aut}(C)$.

So we can apply the following

Lemma 2.5. [Vez Given an exact sequence of algebraic groups over $\mathbb{C}$

$$
1 \longrightarrow H \stackrel{\phi}{\longrightarrow} G \stackrel{\psi}{\longrightarrow} F \longrightarrow 1 .
$$

with $F$ finite and $H$ normal in $G$, we have

$$
A_{G}^{*} \otimes \mathbb{Q} \cong\left(A_{H}^{*} \otimes \mathbb{Q}\right)^{F}
$$

and conclude that

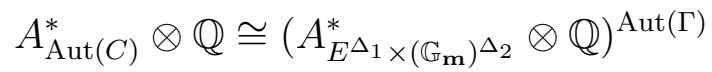

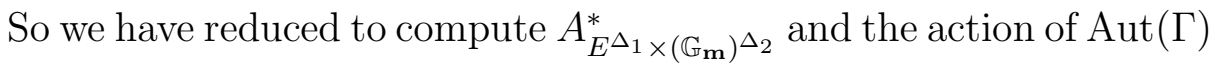
on it.

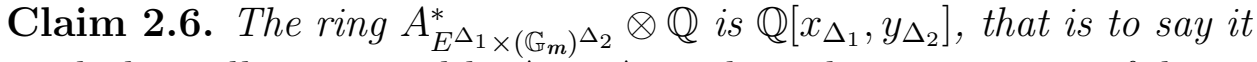
is algebraically generated by $\Delta_{1} \cup \Delta_{2}$ independent generators of degree 1 .

We use the following fact ([Vez] Proposition 2.8): 
Lemma 2.7. For every linear algebraic group $G$, we have

$$
A_{G \times \mathbb{G}_{m}}^{*} \cong A_{G}^{*} \otimes_{\mathbb{Z}} A_{\mathbb{G}_{m}}^{*} .
$$

By recalling that $A_{\mathbb{G}_{\mathbf{m}}}^{*} \otimes \mathbb{Q}$ is isomorphic to $\mathbb{Q}[r]$, where $r$ is an order one class, we have

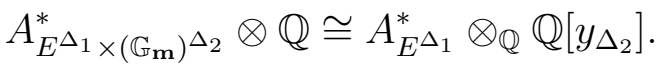

Using the fact that the group $E$ is the semidirect product

$$
0 \longrightarrow \mathbb{G}_{\mathbf{a}} \stackrel{\varphi}{\longrightarrow} E \underset{\rho}{\stackrel{\psi}{\longrightarrow}} \mathbb{G}_{\mathbf{m}} \longrightarrow 1
$$

we can explicit that

$$
A_{E^{\Delta_{1}}}^{*} \otimes \mathbb{Q} \cong \mathbb{Q}\left[x_{\Delta_{1}}\right]
$$

Now we describe the action of $\operatorname{Aut}(\Gamma)$ on

$$
A_{E^{\Delta_{1} \times \mathbb{G}_{\mathbf{m}}}}^{*} \cong \mathbb{Q}\left[x_{\Delta_{1}}, y_{\Delta_{2}}\right] .
$$

In order to do this we need a more explicit description of the classes $x_{\Delta_{1}}, y_{\Delta_{2}}$ through the equivalence (1)

$$
\left(\mathcal{M}_{0,1}^{0}\right)^{\Delta_{1}} \times\left(\mathcal{M}_{0,2}^{0}\right)^{\Delta_{2}} \times\left(\mathcal{M}_{0,3}^{0}\right)^{\Delta_{3}} \cong \widetilde{\mathfrak{M}}_{0}^{\Gamma}
$$

Since $\mathcal{M}_{0,3}^{0} \simeq$ Spec $\mathbb{C}$ we have

$$
\left(\mathcal{M}_{0,1}^{0}\right)^{\Delta_{1}} \times\left(\mathcal{M}_{0,2}^{0}\right)^{\Delta_{2}} \stackrel{\phi}{\rightarrow} \mathfrak{M}_{0}^{\Gamma}
$$

Moreover we have

$$
\begin{aligned}
& \mathcal{M}_{0,1}^{0} \simeq \mathrm{BE} \\
& \mathcal{M}_{0,2}^{0} \simeq \mathrm{B} \mathbb{G}_{\mathbf{m}} .
\end{aligned}
$$

Let $\alpha$ be a vertex of $\Gamma$ such that $e(\alpha)=1$ or 2 . On the component $\mathcal{M}_{0, e(\alpha)}^{0}$ let us consider the universal curve

$$
\mathcal{C}^{\alpha} \stackrel{\widetilde{\Pi}}{\rightarrow} \mathcal{M}_{0, e(\alpha)}^{0} \cdot
$$

On $\mathbb{P}_{\mathbb{C}}^{1}$ we fix coordinates and we define the points

$$
\begin{aligned}
z_{\infty} & :=[1,0] \\
z_{0} & :=[0,1]
\end{aligned}
$$

We can write

- $\mathcal{C}^{\alpha} \simeq\left[\mathbb{P}_{\mathbb{C}}^{1} / E\right]$ when $e(\alpha)=1$

- $\mathcal{C}^{\alpha} \simeq\left[\mathbb{P}_{\mathbb{C}}^{1} / \mathbb{G}_{\mathbf{m}}\right]$ when $e(\alpha)=2$ 
Let us consider on $\mathbb{P}_{\mathbb{C}}^{1}$ the linear bundles $\mathcal{O}\left(z_{\infty}\right)$ and $\mathcal{O}\left(z_{0}\right)$. We have a natural action of $\mathbb{G}_{\mathbf{m}}$ on global sections of both of them induced by the action of $\mathbb{G}_{\mathbf{m}}$ (that, we recall, fixes $z_{\infty}$ and $z_{0}$ ) on $\mathbb{P}_{\mathbb{C}}^{1}$. Similarly we have an action of the group $E$ on global sections of $\mathcal{O}\left(z_{\infty}\right)$. When $e(\alpha)=1$ set

$$
\psi_{\infty, \alpha}^{1}:=c_{1}^{E}\left(H^{0}\left(\mathcal{O}\left(z_{\infty}\right), \mathbb{P}_{\mathbb{C}}^{1}\right)\right)
$$

while, if $e(\alpha)=2$ set

$$
\begin{aligned}
\psi_{\infty, \alpha}^{2} & :=c_{1}^{\mathbb{G}_{\mathbf{m}}}\left(H^{0}\left(\mathcal{O}\left(z_{\infty}\right), \mathbb{P}_{\mathbb{C}}^{1}\right)\right) \\
\psi_{0, \alpha}^{2} & :=c_{1}^{\mathbb{G}_{\mathbf{m}}}\left(H^{0}\left(\mathcal{O}\left(z_{0}\right), \mathbb{P}_{\mathbb{C}}^{1}\right)\right) .
\end{aligned}
$$

Clearly, when $e(\alpha)=2$, we have

$$
\psi_{\infty, \alpha}^{2}+\psi_{0, \alpha}^{2}=0
$$

Now we define

$$
\begin{aligned}
& t_{\alpha}:=\psi_{\infty, \alpha}^{1} \text { when } e(\alpha)=1 \\
& r_{\alpha}:=\psi_{\infty, \alpha}^{2} \text { when } e(\alpha)=2
\end{aligned}
$$

We can write for each $\alpha$ such that $e(\alpha)=2$

$$
r_{\alpha}=\frac{\psi_{\infty, \alpha}^{2}-\psi_{0, \alpha}^{2}}{2}
$$

Clearly all the classes $t_{\Delta_{1}}$ and $r_{\Delta_{2}}$ are of order one and independent. From what we have seen above these classes generates the ring $A^{*}\left(\widetilde{\mathfrak{M}}_{0}^{\Gamma}\right) \otimes \mathbb{Q}$ and we have

$$
A^{*}\left(\widetilde{\mathfrak{M}_{0}^{\Gamma}}\right) \otimes \mathbb{Q} \simeq \mathbb{Q}\left[t_{\Delta_{1}}, r_{\Delta_{2}}\right]
$$

Now we can describe the action of Aut $\Gamma$ on $\mathbb{Q}\left[t_{\Delta_{1}}, r_{\Delta_{2}}\right]$. An element $g \in \operatorname{Aut}(\Gamma)$ acts on $C$ with a permutation $g_{1}$ on the components with one node and a permutation $g_{2}$ of components with two nodes. As we have chosen coordinates on $C_{0}$ such that $\infty$ corresponds to the node of the terminal components, we make $g_{1}$ act directly to the set $\left\{t_{\Delta_{1}}\right\}$. We make $g_{2}$ act similarly on the set $\left\{r_{\Delta_{2}}\right\}$ but we have in addition to consider the sign, that is to say that when $g_{2}$ sends a vertex $P$ of $\Gamma$ to another vertex $\beta$ (such that $e(\alpha)=e(\beta)=2$ ), we have two possibilities

- the automorphism $g$ exchange coordinates 0 and $\infty$ and so we have

$$
g\left(r_{\alpha}\right)=g\left(\frac{\psi_{\infty, \alpha}^{2}-\psi_{0, \alpha}^{2}}{2}\right)=\frac{\psi_{0, \beta}^{2}-\psi_{\infty, \beta}^{2}}{2}=-r_{\beta}
$$

- the automorphism $g$ sends 0 in 0 and $\infty$ in $\infty$; in this case we have

$$
g\left(r_{\alpha}\right)=r_{\beta}
$$


Definition 2.8. We define $\gamma_{i}$ as the class in $A^{*}\left(\mathfrak{M}_{0}^{\leq i}\right)$ of $\mathfrak{M}_{0}^{i}$. We will indicate with $\gamma_{i} \in A^{*}\left(\mathfrak{M}_{0}\right)$ also the class of the closure of $\mathfrak{M}_{0}^{i}$ in $\mathfrak{M}_{0}$. Similarly we define $\gamma_{\Gamma}$ as the class of the closure of $\mathfrak{M}_{0}^{\Gamma}$ in $\mathfrak{M}_{0}$.

Proposition 2.9. Let $\Gamma$ be a tree of maximal multiplicity at most three (except the single point) and $C$ be the curve of topological type $\Gamma$. Let us consider the étale covering

$$
\widetilde{\mathfrak{M}}_{0}^{\Gamma} \stackrel{\phi}{\rightarrow} \mathfrak{M}_{0}^{\Gamma}
$$

Let $C_{\Gamma}$ be the components of $C$ (which we see as vertex of $\Gamma$ ). Let $E(\Gamma)$ be the set of edges. If $(\alpha, \beta) \in E(\Gamma)$ we call $z_{\alpha \beta}$ the common point of $C_{\alpha}$ and $C_{\beta}$. Then, by following notation of Proposition 2.4, we have

$$
\phi^{*} \gamma_{\Gamma}=\prod_{(\alpha, \beta) \in E(\Gamma)}\left(\psi_{z_{\alpha \beta} \mid \alpha, \alpha}^{e(\alpha)}+\psi_{\left.z_{\alpha \beta}\right|_{\beta}, \beta}^{e(\alpha)}\right) .
$$

In particular the classes of each stratum of $\mathfrak{M}_{0}^{\leq 3}$ after fixing coordinates on $C$ and ordering components of $\Delta_{1}$ and $\Delta_{2}$, are:

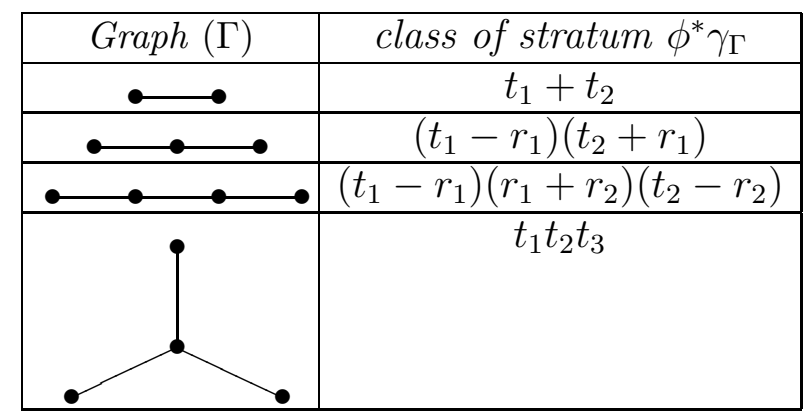

Proof. For every tree $\Gamma$ of maximal multiplicity at most three (except the single point), let us consider the regular embedding

$$
\mathfrak{M}_{0}^{\Gamma}=\operatorname{BAut}(C) \stackrel{\text { in }}{\rightarrow} \mathfrak{M}_{0}^{\leq \delta}
$$

where $\delta$ is the number of edges of $\Gamma$.

Let us consider the normal bundle

$$
N_{\Gamma}:=N_{\mathfrak{M}_{0}^{\Gamma} / \mathfrak{M} \times \delta}^{\delta \delta}
$$

It is known that $N_{\Gamma}=\operatorname{def}_{\Gamma}$ is the space of first order deformations of $\mathfrak{M}_{0}^{\Gamma}$

$$
\bigoplus_{(\alpha, \beta) \in E(\Gamma)} T_{z_{\alpha \beta}}\left(C_{\alpha}\right) \otimes T_{z_{\alpha \beta}}\left(C_{\beta}\right)
$$


As usual let us consider the étale covering

$$
\begin{aligned}
& \widetilde{\mathfrak{M}}_{0}^{\Gamma} \stackrel{\phi}{\rightarrow} \mathfrak{M}_{0}^{\Gamma} \\
& \mathrm{BH} \rightarrow \mathrm{B}(\operatorname{Aut}(\Gamma) \ltimes H)
\end{aligned}
$$

and set $\widetilde{N}_{\Gamma}=\phi^{*} N_{\Gamma}$. We notice that the point $z_{\alpha \beta}$ on each component $C_{\alpha}$ (which we call $\left.z_{\alpha \beta}\right|_{\alpha}$ ) is 0,1 or $\infty$.

By using notation of Proposition 2.4 we have on $\widetilde{\mathfrak{M}}_{0}^{\Gamma}$

$$
c_{1}^{G_{\alpha}}\left(T_{z_{\alpha \beta}}\left(C_{\alpha}\right)\right)=\psi_{\left.z_{\alpha \beta}\right|_{\alpha}, \alpha}^{e(\alpha)}
$$

where $G_{\alpha}=E$ if $e(\alpha)=1, G_{\alpha}=\mathbb{G}_{\mathbf{m}}$ if $e(\alpha)=2$ and $G_{\alpha}=$ id if $e(\alpha)=3$ Notice that $c_{1}^{G_{\alpha}}\left(T_{z_{\alpha \beta}}\right.$ is zero when $e(\alpha)=3$, consequently

$$
c_{\text {top }}^{H}\left(\widetilde{N}_{\Gamma}\right)=\prod_{(\alpha, \beta) \in E(\Gamma)}\left(\psi_{\left.z_{\alpha \beta}\right|_{\alpha}, \alpha}^{e(\alpha)}+\psi_{\left.z_{\alpha \beta}\right|_{\beta}, \beta}^{e(\beta)}\right) .
$$

We have the following relation in $A^{*}\left(\mathfrak{M}_{0}^{\Gamma}\right)$

$$
i n^{*} i n_{*}\left[\mathfrak{M}_{0}^{\Gamma}\right]=c_{t o p}^{H}\left(N_{\Gamma}\right) \cap\left[\mathfrak{M}_{0}^{\Gamma}\right]
$$

and so

$$
\phi^{*} \gamma_{\Gamma}=c_{\text {top }}^{H}\left(\widetilde{N}_{\Gamma}\right)
$$

Remark 2.10. Given a choice of coordinates the class $\phi^{*} \gamma_{\Gamma}$ is invariant for the action of $\operatorname{Aut}(\Gamma)$ given in Proposition [2.4, so we actually can see it as the class $\gamma_{\Gamma}$ in $\mathfrak{M}_{0}^{\Gamma}$.

Moreover we have shown that these classes are not 0 -divisor in the ring $A^{*}\left(\mathfrak{M}_{0}^{\Gamma}\right)$ for each $\Gamma$ corresponding to a stratum in $\mathfrak{M}_{0}^{\leq 3}$.

This fails if we consider more than four nodes. For example if we consider the following graph $\Gamma$

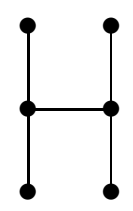

we have $\phi^{*} \gamma_{\Gamma}=0$.

For future reference we can now state the following

Proposition 2.11. The Chow ring $A^{*}\left(\mathfrak{M}_{0}^{\leq 3}\right) \otimes \mathbb{Q}$ injects into the product of $A^{*}\left(\mathfrak{M}_{0}^{\Gamma}\right) \otimes \mathbb{Q}$ over trees with at most three edges. 
Proof. From Proposition 2.9 and Remark (2.10), we have, for each $\delta \leq 3$, the following exact sequence of additive groups

$$
0 \rightarrow A^{*}\left(\mathfrak{M}_{0}^{\delta}\right) \otimes \mathbb{Q} \stackrel{i_{*}^{\delta}}{\rightarrow} A^{*}\left(\mathfrak{M}_{0}^{\leq \delta}\right) \otimes \mathbb{Q} \stackrel{j^{* \delta}}{\rightarrow} A^{*}\left(\mathfrak{M}_{0}^{\leq(\delta-1)}\right)
$$

where

$$
\begin{aligned}
& i^{\delta}: \mathfrak{M}_{0}^{\delta} \rightarrow \mathfrak{M}_{0}^{\leq \delta} \\
& j^{\delta}: \mathfrak{M}_{0}^{\leq(\delta-1)} \rightarrow \mathfrak{M}_{0}^{\leq \delta}
\end{aligned}
$$

are the natural closed embeddings.

Let us consider the morphism

$$
A^{*}\left(\mathfrak{M}_{0}^{\leq 3}\right) \otimes \mathbb{Q} \stackrel{\psi}{\rightarrow} \prod_{\delta=0}^{3} A^{*}\left(\mathfrak{M}_{0}^{\delta}\right) \otimes \mathbb{Q}
$$

as the product of the maps $i^{3 *}, i^{2 *} j^{3 *}, i^{1 *} j^{2 *} j^{3 *}$ and $j^{1 *} j^{2 *} j^{3 *}$. Let $a$ be an element of $A^{*}\left(\mathfrak{M}_{0}^{\leq 3}\right) \otimes \mathbb{Q}$ different from zero. If $\psi(a)$ is zero then it cannot be in the image of $i_{*}^{3}$ consequently $j^{3 *}(a) \in A^{*}\left(\mathfrak{M}_{0}^{\leq 2}\right)$ is different from zero. We can continue till we obtain that $j^{1 *} j^{2 *} j^{3 *}$ is different from zero: absurd.

\section{Restriction of Classes to strata}

Let us consider two trees $\Gamma$ and $\Gamma^{\prime}$ with maximal multiplicity at most 3 and number of edges respectively equal to $\delta$ and $\delta^{\prime}$.

Definition 3.1. Given two graphs as above we call an ordered deformation of $\Gamma$ into $\Gamma^{\prime}$ any surjective map of vertices $d: \Gamma^{\prime} \rightarrow \Gamma$ such that

(1) for each $P, Q \in \Gamma^{\prime}$ we have $d(P)=d(Q)=A \in \Gamma^{\prime}$ only if for each $R$ in the connected path from $P$ to $Q$ we have $d(R)=A$;

(2) for each edge $(P, Q) \in \Gamma^{\prime}$ such that $d(P) \neq d(Q)$ there must be an edge in $\Gamma$ between $d(P)$ and $d(Q)$.

We denote by $\operatorname{def}_{o}\left(\Gamma, \Gamma^{\prime}\right)$ the set of deformations.

Example 3.2. Let $\Gamma$ and $\Gamma^{\prime}$ be the following graphs

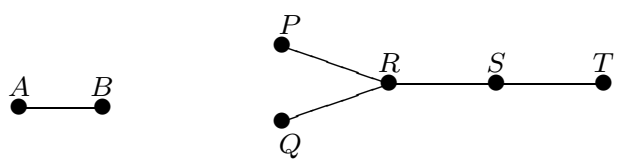

we have the following 8 ordered deformations

\begin{tabular}{|ll|ll|}
\hline 1$)(P, R, S, T) \mapsto A$ & $Q \mapsto B$ & $5) Q \mapsto A$ & $(P, R, S, T) \mapsto B$ \\
\hline 2$)(Q, R, S, T) \mapsto A$ & $P \mapsto B$ & $6) P \mapsto A$ & $(Q, R, S, T) \mapsto B$ \\
\hline 3$)(P, Q, R) \mapsto A$ & $(S, T) \mapsto B$ & $7)(S, T) \mapsto A$ & $(P, Q, R) \mapsto B$ \\
\hline 4$)(P, Q, R, S) \mapsto A$ & $T \mapsto B$ & $8) T \mapsto A$ & $(P, Q, R, S) \mapsto B$ \\
\hline
\end{tabular}


There exist two different equivalence relations in $\operatorname{def}_{o}\left(\Gamma, \Gamma^{\prime}\right)$. We say that two elements $d_{1}, d_{2}$ are in $\sim_{\Gamma}$ if there exists a $\gamma \in \operatorname{Aut}(\Gamma)$ such that $d_{2}=\gamma d_{1}$. Similarly we say that two elements $d_{1}, d_{2}$ are in $\sim_{\Gamma^{\prime}}$ if there exists a $\gamma^{\prime} \in \operatorname{Aut}\left(\Gamma^{\prime}\right)$ such that $d_{2}=d_{1} \gamma^{\prime}$.

Definition 3.3. We call $\Gamma$-deformations (or simply deformations) from $\Gamma$ to $\Gamma^{\prime}$ the set

$$
\operatorname{def}_{\Gamma}\left(\Gamma, \Gamma^{\prime}\right):=\operatorname{def}_{o}\left(\Gamma, \Gamma^{\prime}\right) / \sim_{\Gamma} .
$$

We call $\Gamma^{\prime}$-deformations from $\Gamma$ to $\Gamma^{\prime}$ the set

$$
\operatorname{def}_{\Gamma^{\prime}}\left(\Gamma, \Gamma^{\prime}\right):=\operatorname{def}_{o}\left(\Gamma, \Gamma^{\prime}\right) / \sim_{\Gamma^{\prime}} .
$$

In the above example we take as representatives of $\Gamma$-deformations the first 4 ordered deformations. On the other hand we have

$$
1 \sim_{\Gamma^{\prime}} 2 \quad 5 \sim_{\Gamma^{\prime}} 6
$$

From topological arguments we have the following

Proposition 3.4. Let $C \stackrel{\pi}{\rightarrow} T$ be a family of rational nodal curves over an irreducible scheme T. Suppose further that the generic fiber has topological type $\Gamma$, then there exists a fiber of topological type $\widehat{\Gamma}$ only if there exists an ordered deformation of $\Gamma$ into $\widehat{\Gamma}$.

Now let us consider the étale map

$$
\widetilde{\mathfrak{M}_{0}^{\Gamma}} \stackrel{\phi}{\rightarrow} \mathfrak{M}_{0}^{\Gamma}
$$

We have given above a description of $A^{*} \mathfrak{M}_{0}$ as the subring of polynomials of $A^{*}\left(\widetilde{\mathfrak{M}}_{0}^{\Gamma}\right)$ in the classes (corresponding to sections of $\widetilde{\mathfrak{M}}_{0}^{\Gamma}$ ) $t_{1}, \ldots, t_{\delta_{1}}, r_{1}, \ldots, r_{\delta_{2}}$ invariant for the action of $\operatorname{Aut}(\Gamma)$.

We call $\mathcal{M}_{0, i}$ the stack of rational nodal curves with $i$ sections. Let $\left(\widetilde{\mathfrak{M}}_{0}^{\Gamma}\right)^{\leq \delta^{\prime}-\delta}$ be the substack of

$$
\left(\mathcal{M}_{0,1}\right)^{\Delta_{1}} \times\left(\mathcal{M}_{0,2}\right)^{\Delta_{2}} \times\left(\mathcal{M}_{0,3}\right)^{\Delta_{3}}
$$

whose fibers have at most $\delta^{\prime}-\delta$ nodes (the sum of nodes is taken over all the connected components). Polynomials in $\mathbb{Q}\left[t_{1}, \ldots, t_{\delta_{1}}, r_{1}, \ldots, r_{\delta_{2}}\right]$ has a natural extension to $\left(\widetilde{\mathfrak{M}}_{0}^{\Gamma}\right)^{\leq \delta^{\prime}-\delta}$. Let us fix one of such polynomials $a$ which are invariants for the action of Aut $\Gamma$.

The étale covering

$$
\widetilde{\mathfrak{M}}_{0}^{\Gamma} \stackrel{\phi}{\rightarrow} \mathfrak{M}_{0}^{\Gamma}
$$

is obtained by gluing sections in a way which depends on $\Gamma$. 
By gluing sections in the same way we obtain a functor

$$
\left(\widetilde{\mathfrak{M}}_{0}^{\Gamma}\right)^{\leq \delta^{\prime}-\delta} \stackrel{\Pi}{\rightarrow} \mathfrak{M}_{0}^{\delta^{\prime}}
$$

Corollary 3.5. With the above notation we have that the closure $\overline{\mathfrak{M}}_{0}^{\Gamma}$ of $\mathfrak{M}_{0}^{\Gamma}$ in $\mathfrak{M}_{0}^{\leq \delta^{\prime}}$ is

$$
\Pi\left(\left(\widetilde{\mathfrak{M}}_{0}^{\Gamma}\right)^{\leq \delta^{\prime}-\delta}\right)
$$

Proof. From Proposition 3.4 we have

$$
\overline{\mathfrak{M}}_{0}^{\Gamma} \subseteq \Pi\left(\left(\widetilde{\mathfrak{M}}_{0}^{\Gamma}\right)^{\leq \delta^{\prime}-\delta}\right)
$$

On the other hand let $C_{\Omega} \stackrel{\pi}{\rightarrow}$ Spec $\Omega$ be the image in $\mathfrak{M}_{0}^{\delta^{\prime}}$ of a geometric point of $\left(\widetilde{\mathfrak{M}}_{0}^{\Gamma}\right)^{\leq \delta^{\prime}-\delta}$. The dual graph $\Gamma_{\Omega}$ of $C_{\Omega}$ is a deformation of $\Gamma$. In order to show that $C_{\Omega} \stackrel{\pi}{\rightarrow} \operatorname{Spec} \Omega$ is a geometric point of $\overline{\mathfrak{M}}_{0}^{\Gamma}$, we fix a deformation $d: \Gamma_{\Omega} \rightarrow \Gamma$. For each vertex $A$ of $\Gamma$, the set $d^{-1}(A)$ is a subtree of $\Gamma_{\Omega}$. We can give a deformation $C_{A} \stackrel{\pi}{\rightarrow} T$ of $C_{\Omega}$ such that the generic fiber is $\mathbb{P}^{1}$. Furthermore we can define on $C_{A} \stackrel{\pi}{\rightarrow} T$ a family of $E(A)$ that respect $d$. At last we glue all $C_{A}$ along sections and obtain a deformation $C \stackrel{\pi}{\rightarrow} T$ of $C_{\Omega} \stackrel{\pi}{\rightarrow} \operatorname{Spec} \Omega$ in $\overline{\mathfrak{M}}_{0}^{\Gamma}(\operatorname{Spec} \Omega)$.

Definition 3.6. We call the image of $\Pi$ :

$$
\mathfrak{M}_{0}^{\operatorname{def}\left(\Gamma, \delta^{\prime}\right)}:=\Pi\left(\left(\widetilde{\mathfrak{M}}_{0}^{\Gamma}\right)^{\leq \delta^{\prime}-\delta}\right) \subset \mathfrak{M}_{0}^{\leq \delta^{\prime}}
$$

Proposition 3.7. The map

$$
\Pi:\left(\widetilde{\mathfrak{M}}_{0}^{\Gamma}\right)^{\delta^{\prime}-\delta} \rightarrow \mathfrak{M}_{0}^{<\delta^{\prime}}
$$

is finite.

Proof. (sketch) We have to prove that $\Pi$ is representable, with finite fibers and proper. The not trivial property to verify is properness. We can prove it through the valutative criterion (see [Hrt] p.101).

Therefore the map $\Pi$ is finite hence projective, so we have the pushforward Kre

$$
\Pi_{*}: A^{*}\left(\left(\widetilde{\mathfrak{M}}_{0}^{\Gamma}\right)^{\delta^{\prime}-\delta}\right) \rightarrow A^{*}\left(\mathfrak{M}_{0}^{\operatorname{def}\left(\Gamma, \delta^{\prime}\right)}\right)
$$


Definition 3.8. Let $\Gamma$ be a tree with maximal multiplicity $\leq 3$ and $\Gamma^{\prime}$ a deformation of $\Gamma$ (with maximal multiplicity $\leq 3$ ) with $\delta^{\prime}$ edges. Let $a$ be a class in $A^{*}\left(\mathfrak{M}_{0}^{\Gamma}\right) \otimes \mathbb{Q}$ and $\tilde{a}$ its $\delta^{\prime}$-lifting. With reference to the cartesian diagram

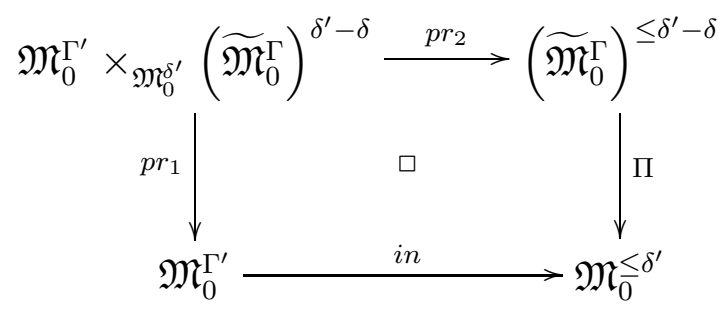

we define

$$
\Psi\left(\Gamma, \Gamma^{\prime}\right):=\mathfrak{M}_{0}^{\Gamma^{\prime}} \times_{\mathfrak{M}_{0}^{\delta^{\prime}}}\left(\widetilde{\mathfrak{M}}_{0}^{\Gamma}\right)^{\delta^{\prime}-\delta} .
$$

Topological arguments show the following

Proposition 3.9. $\Psi\left(\Gamma, \Gamma^{\prime}\right)$ is a disjoint union of components that we write as

$$
\Psi\left(\Gamma, \Gamma^{\prime}\right)=: \coprod_{\xi \in \operatorname{def} f_{\Gamma^{\prime}}\left(\Gamma, \Gamma^{\prime}\right)} \Psi\left(\Gamma, \Gamma^{\prime}\right)_{\xi}
$$

where the union is taken over the set of ordered deformations up to $\sim_{\Gamma^{\prime}}$.

\section{Example 3.10.}

Let $\Gamma$ and $\Gamma^{\prime}$ be the graphs of the Example 3.2. We have $\delta^{\prime}-\delta=3$ and

with a double covering

$$
\widetilde{\mathfrak{M}}_{0}^{\Gamma}=\mathcal{M}_{0,1}^{0} \times \mathcal{M}_{0,1}^{0}
$$

$$
\widetilde{\mathfrak{M}}_{0}^{\Gamma} \stackrel{\phi}{\rightarrow} \mathfrak{M}_{0}^{\Gamma}
$$

Clearly $\Psi\left(\Gamma, \Gamma^{\prime}\right)$ is an inclusion of components in

$$
\coprod_{i, j: i+j=3}\left(\mathcal{M}_{0,1}^{i} \times \mathcal{M}_{0,1}^{j}\right)
$$

We have that $\Psi\left(\Gamma, \Gamma^{\prime}\right)$ has 6 components which corresponds to deformations (enumerated in Example 3.2) up to $\sim_{\Gamma^{\prime}}$, with the following inclusions:

- deformation 1 (which is $\Gamma^{\prime}$-equivalent to 2 ) and 4 correspond to two connected components of $\mathcal{M}_{0,1}^{3} \times \mathcal{M}_{0,1}^{0}$

- deformation 3 corresponds to a connected component of $\mathcal{M}_{0,1}^{2} \times$ $\mathcal{M}_{0,1}^{1}$

- deformation 7 corresponds to a connected components of $\mathcal{M}_{0,1}^{1} \times$ $\mathcal{M}_{0,1}^{2}$ 
- deformation 5 (which is $\Gamma^{\prime}$-equivalent to 6 ) and 8 corresponds to two connected components of $\mathcal{M}_{0,1}^{0} \times \mathcal{M}_{0,1}^{3}$

Definition 3.11. Let us consider a class $a$ in $A^{*}\left(\mathfrak{M}_{0}^{\Gamma}\right) \otimes \mathbb{Q}$. With reference to the étale covering

$$
\widetilde{\mathfrak{M}_{0}^{\Gamma}} \stackrel{\phi}{\rightarrow} \mathfrak{M}_{0}^{\Gamma}
$$

we define the lifting of $a$

$$
\widetilde{a}:=\frac{\phi^{*} a}{\sigma} \in A^{*}\left(\widetilde{\mathfrak{M}}_{0}^{\Gamma}\right) \otimes \mathbb{Q},
$$

this means that $\phi_{*}(\widetilde{a})=a$. Since $\widetilde{a}$ can be written as a polynomial in the classes $\psi$ defined in Proposition 2.4 that depends only on $\Gamma$, it has a natural extension to $A^{*}\left(\left(\widetilde{\mathfrak{M}}_{0}^{\Gamma}\right)^{\delta^{\prime}-\delta}\right) \otimes \mathbb{Q}$ that we call $\delta^{\prime}$-lifting of $a$ and we still write $\tilde{a}$.

For every $\xi \in \operatorname{def}_{\Gamma^{\prime}}\left(\Gamma, \Gamma^{\prime}\right)$ we have the related commutative diagram

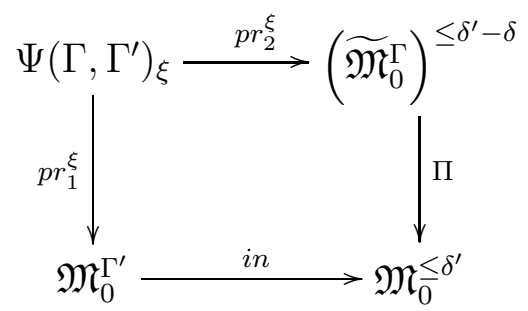

The map $p r_{2}^{\xi}$ is a closed immersion of codimension $\delta^{\prime}-\delta$. Let us still call $\widetilde{a}$ the pullback of the polynomial $\widetilde{a}$ through $p r_{2}^{\xi}$. By the excess intersection formula (Section 6.3 [Fulton], similar arguments show it for algebraic stacks) we have

$$
i n^{!}(\widetilde{a})=\sum_{\xi \in \operatorname{def}_{\Gamma^{\prime}}\left(\Gamma, \Gamma^{\prime}\right)}\left(\widetilde{a} \cdot c_{t o p}\left[\mathcal{N}^{\xi}\right]\right) .
$$

where $\mathcal{N}^{\xi}:=\left(p r_{1}^{\xi *} \mathcal{N}_{i n}\right) / \mathcal{N}_{p r}^{\xi}$

For each $\xi \in \operatorname{def}_{\Gamma^{\prime}}\left(\Gamma, \Gamma^{\prime}\right)$ we have an étale covering

$$
f_{\xi}: \widetilde{\mathfrak{M}}^{\Gamma^{\prime}} \rightarrow \Psi\left(\Gamma, \Gamma^{\prime}\right)_{\xi}
$$

that glue along sections.

For every $\xi \in \operatorname{def}_{\Gamma^{\prime}}\left(\Gamma, \Gamma^{\prime}\right)$ let us call $\sigma_{\xi}^{\prime}$ the degree of $p r_{1}^{\xi}$. 
We have the following commutative diagram

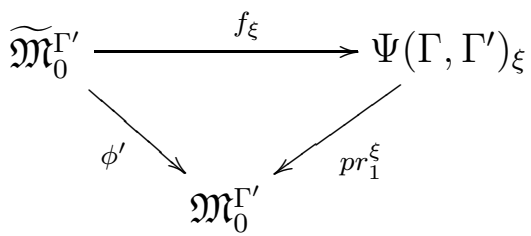

from which we have

$$
\operatorname{ord} \phi^{\prime}=\operatorname{ord} f_{\xi} \cdot \operatorname{ord} \sigma_{\xi}^{\prime}
$$

Remark 3.12. The order of $f_{\xi}$ is the number of $g \in \operatorname{Aut}\left(\Gamma^{\prime}\right)$ such that for each deformation $d$ associated to $\xi$ the deformation $d \circ g$ is $\Gamma^{\prime}$-equivalent to $d$.

We want to explicit $\phi^{\prime *} \operatorname{pr}_{1 *} i n^{!}(\widetilde{a})$ in $A^{*}\left(\widetilde{\mathfrak{M}}_{0}^{\Gamma^{\prime}}\right) \otimes \mathbb{Q}$ : being invariant for the action of $\operatorname{Aut}\left(\Gamma^{\prime}\right)$ we can see it as a class in $\mathfrak{M}_{0}^{\Gamma^{\prime}}$ which is the restriction of the extension of the class $a \in A^{*}\left(\mathfrak{M}_{0}^{\Gamma}\right) \otimes \mathbb{Q}$.

Proposition 3.13. We have

$$
\phi^{\prime *} p r_{1 *} i n^{!}(\widetilde{a})=\sum_{\xi \in \operatorname{def}_{\Gamma^{\prime}}\left(\Gamma, \Gamma^{\prime}\right)} \sigma_{\xi}^{\prime} f_{\xi}^{*}(\widetilde{a}) \cdot c_{t o p}\left(\widetilde{\mathcal{N}}^{\xi}\right)
$$

where

$$
\tilde{\mathcal{N}}^{\xi}:=f_{\xi}^{*} \mathcal{N}^{\xi} .
$$

Proof. Putting together all the above remarks and definitions we have

$$
\begin{aligned}
\phi^{\prime *} p r_{1 *} i n^{!}(\widetilde{a}) & =\phi^{\prime *} p r_{1 *} \sum_{\xi \in \operatorname{def}_{\Gamma^{\prime}}\left(\Gamma, \Gamma^{\prime}\right)}\left(\widetilde{a} \cdot c_{t o p}\left[\mathcal{N}^{\xi}\right]\right) \\
& =\phi^{\prime *} \sum_{\xi \in \operatorname{def}_{\Gamma^{\prime}}\left(\Gamma, \Gamma^{\prime}\right)} p r_{1 *}^{\xi}\left(\widetilde{a} \cdot c_{t o p}\left[\mathcal{N}^{\xi}\right]\right) \\
& =\sum_{\xi \in \operatorname{def}_{\Gamma^{\prime}}\left(\Gamma, \Gamma^{\prime}\right)} f_{\xi}^{*} p r_{1}^{\xi *} p r_{1 *}^{\xi}\left(\widetilde{a} \cdot c_{t o p}\left[\mathcal{N}^{\xi}\right]\right) \\
& =\sum_{\xi \in \operatorname{def}_{\Gamma^{\prime}}\left(\Gamma, \Gamma^{\prime}\right)} \sigma_{\xi}^{\prime} f_{\xi}^{*}(\widetilde{a}) \cdot c_{t o p}\left(\widetilde{\mathcal{N}}^{\xi}\right)
\end{aligned}
$$

We explicit now the computation of classes corresponding to strata.

Proposition 3.14. Given a tree $\Gamma$ with maximal multiplicity $\leq 3$, let $\gamma_{\Gamma}$ be the class of $\overline{\mathfrak{M}}_{0}^{\Gamma}$ in $\mathfrak{M}_{0}$ and let $\Gamma^{\prime}$ be another tree with maximal multiplicity $\leq 3$. 
If $\Gamma^{\prime}$ is a deformation of $\Gamma$, then the restriction of $\gamma_{\Gamma}$ to $A^{*}\left(\mathfrak{M}^{\Gamma^{\prime}}\right) \otimes \mathbb{Q}$ is (following the above notation)

$$
\sum_{\xi \in \operatorname{def}_{\Gamma^{\prime}}\left(\Gamma, \Gamma^{\prime}\right)} \frac{\sigma_{\xi}^{\prime}}{\sigma} c_{t o p}\left(\widetilde{\mathcal{N}}^{\xi}\right) .
$$

If $\Gamma^{\prime}$ is not a deformation of $\Gamma$ then the restriction is 0 .

Proof. In case $\Gamma^{\prime}$ is a deformation of $\Gamma$, the polynomial $a$ of Proposition (3.13) is 1 , consequently $\widetilde{a}$ is $1 / \sigma$. With reference to the étale covering $\widetilde{\mathfrak{M}}_{0}^{\Gamma^{\prime}} \stackrel{\phi}{\rightarrow} \mathfrak{M}_{0}^{\Gamma^{\prime}}$ and the inclusion $\mathfrak{M}_{0}^{\Gamma^{\prime}} \stackrel{\text { in }}{\rightarrow} \mathfrak{M}_{0}^{\delta^{\prime}}$, from Proposition 3.13 we obtain

$$
\sum_{\xi \in \operatorname{def}_{\Gamma^{\prime}}\left(\Gamma, \Gamma^{\prime}\right)} \frac{\sigma_{\xi}^{\prime}}{\sigma} c_{t o p}\left(\widetilde{\mathcal{N}}^{\xi}\right) .
$$

If $\Gamma^{\prime}$ is not a deformation of $\Gamma$ then using Corollary 3.5 and basic topological arguments, we have that $\mathfrak{M}_{0}^{\Gamma^{\prime}}$ does not intersect the closure of $\mathfrak{M}_{0}^{\Gamma}$ in $\mathfrak{M}_{0}$, so the restriction of the class must be 0 .

Remark 3.15. For each $\xi \in \operatorname{def}_{\Gamma^{\prime}}\left(\Gamma, \Gamma^{\prime}\right)$, we have an exact sequence of sheaves

$$
0 \rightarrow f_{\xi}^{*} \mathcal{N}_{p r_{2}^{\xi}} \rightarrow \phi^{\prime *} \mathcal{N}_{i n} \rightarrow \tilde{\mathcal{N}}^{\xi} \rightarrow 0
$$

We have seen in Proposition 2.9 how to compute $c_{t o p}\left(\phi^{\prime *} \mathcal{N}_{i n}\right)$, similarly we can compute $c_{\text {top }}\left(f_{\xi}^{*} \mathcal{N}_{p r_{2}^{\xi}}\right)$ and finally we consider the following relation (that follows from the exact sequence)

$$
c_{\text {top }}\left(\phi^{\prime *} \mathcal{N}_{i n}\right)=c_{t o p}\left(f_{\xi}^{*} \mathcal{N}_{p r_{2}^{\xi}}\right) \cdot c_{t o p}\left(\widetilde{\mathcal{N}}^{\xi}\right) .
$$

We carry on the calculation for trees with at most three nodes in the last Section.

\section{MUMFORD CLASSES}

Given a tree $\Gamma$ with at most four vertices, we have the restriction of the dualizing sheaf $\omega_{0}:=\omega_{\mathcal{C} / \mathfrak{M}_{0}}$ on the universal curve $\mathcal{C}^{\Gamma}$ of $\mathfrak{M}_{0}^{\Gamma}$. We call $\omega_{0}^{\Gamma}:=\omega_{\mathcal{C}^{\Gamma} / \mathfrak{M}_{0}^{\Gamma}}$ its restriction.

We consider two kinds of classes on $A_{\text {Aut }\left(C_{0}\right)}^{*}$ induced by the sheaf $\omega_{0}^{\Gamma}$ :

(1) the pushforward of polynomials of the Chern class $K:=c_{1}\left(\omega_{0}^{\Gamma}\right)$;

(2) the Chern classes of the pushforward of $\omega_{0}$.

The first kind will give us the equivalent of Mumford classes, but on $\mathfrak{M}_{0}^{\leq 3}$ we can only define classes of the second type.

The aim of this section is to compute classes of the first kind for $\Gamma$ with at most three nodes and to describe them as polynomials in 
classes of the second kind. If this description is independent from the graph $\Gamma$ then we can define them as elements of $A^{*}\left(\mathfrak{M}_{0}^{\leq 3}\right)$.

First of all we define on $\mathcal{C}^{\Gamma}$ and $\mathfrak{M}_{0}^{\Gamma}$ the following classes

$$
\begin{aligned}
K & :=c_{1}\left(\omega_{0}^{\Gamma}\right) \in A^{1}\left(C^{\Gamma}\right), \\
\kappa_{i} & :=\Pi_{*}\left(K^{i+1}\right) \in A^{i}\left(\mathfrak{M}_{0}^{\Gamma}\right) .
\end{aligned}
$$

Such classes $K_{i}$ (introduced in Mum2 for the moduli spaces of stable curves) are called Mumford classes.

Mumford classes on $\mathfrak{M}_{0}^{0}$

The stack $\mathfrak{M}_{0}^{0}$ is $\mathrm{BP} G l_{2}$.

Let us consider the universal curve

$$
\left[\mathbb{P}_{\mathbb{C}}^{1} / \mathbb{P} G l_{2}\right] \stackrel{\Pi}{\rightarrow} \mathrm{BP} G l_{2} .
$$

We call $\bar{\Pi}$ the induced map $\mathbb{P}_{\mathbb{C}}^{1} \rightarrow$ Spec $\mathbb{C}$ and $\bar{\omega}_{0}^{0}$ a lifting of $\omega_{0}^{0}$ on $\mathbb{P}_{\mathbb{C}}^{1}$.

We notice that $\left(\bar{\omega}_{0}^{0}\right)^{\vee}=T_{\bar{\Pi}}\left(T_{\Pi}\right.$ is the relative tangent bundle along $\Pi$ ), we set

$$
K:=c_{1}^{\mathbb{P} G l_{2}}\left(T_{\bar{\Pi}}\right)=-c_{1}^{\mathbb{P} G l_{2}}\left(\bar{\omega}_{0}^{0}\right) .
$$

Furthermore $\bar{\Pi}_{*}\left(T_{\bar{\Pi}}\right)=H^{0}\left(\mathbb{P}_{\mathbb{C}}^{1}, T_{\bar{\Pi}}\right)=\mathfrak{s l}_{2}$ seen as adjoint representation of $\mathbb{P} G l_{2}$.

By applying the equivariant Grothendieck-Riemann-Roch Theorem we obtain

$$
\begin{aligned}
c h\left(\Pi_{*}\left(\omega_{0}^{\vee}\right)\right) & =\Pi_{*}\left(T d\left(T_{\Pi}\right) \operatorname{ch}\left(\omega_{0}^{\vee}\right)\right) \\
c h^{\mathbb{P} G l_{2}}\left(\mathfrak{s l}_{2}\right) & =\Pi_{*}\left(T d^{\mathbb{P} G l_{2}}\left(T_{\bar{\Pi}}\right) \operatorname{ch}^{\mathbb{P} G l_{2}}\left(T_{\bar{\Pi}}\right)\right) \\
3-c_{2}^{\mathbb{P} G l_{2}}\left(\mathfrak{s l}_{2}\right) & =\Pi_{*}\left[\left(e^{-(K)}\right)\left(\frac{-K}{1-e^{K}}\right)\right]
\end{aligned}
$$

By applying GRR to the trivial linear bundle we obtain:

$$
1=\Pi_{*}\left[\frac{-K}{1-e^{K}}\right]
$$

If we subtract the second equation from the first, we obtain

$$
2-c_{2}^{\mathbb{P} G l_{2}}\left(\mathfrak{s l}_{2}\right)=\Pi_{*}\left[\left(\frac{1-e^{K}}{e^{K}}\right)\left(\frac{-K}{1-e^{K}}\right)\right]=\Pi_{*}\left[-K e^{-K}\right],
$$

from which we get the following

Proposition 4.1. On $\mathfrak{M}_{0}^{0}$ we have

$$
k_{0}=-2, \quad k_{2}=2 c_{2}^{\mathbb{P} G l_{2}}\left(\mathfrak{s l}_{2}\right), \quad \kappa_{1}=k_{3}=0 .
$$




\section{Mumford classes on strata of singular curves}

Now let us consider the following cartesian diagram (see Section 2)

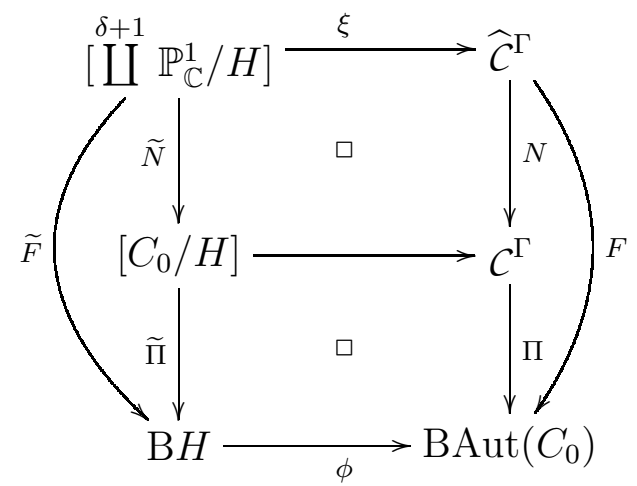

where $N: \widehat{C}^{\Gamma} \rightarrow C^{\Gamma}$ is the normalization of $C^{\Gamma}$.

In the following we call $\widetilde{\omega}_{0}^{\Gamma}$ the sheaf

$$
\xi^{*} N^{*}\left(\omega_{0}^{\Gamma}\right) .
$$

and $\widetilde{K}:=c_{1}\left(\omega_{0}\right)$.

Proposition 4.2. Using the above notation, the Mumford classes $\mathcal{K}_{i} \in$ $A^{i}(B F \ltimes H) \otimes \mathbb{Q}$ are described by the following relation

$$
\phi_{*} \widetilde{F}_{*}\left(\widetilde{K}^{i+1}\right)=\sigma \kappa_{i}
$$

Proof. Let us fix an index $i \in \mathbb{N}$. Since the map $N$ is finite and generically of degree 1 we have $N_{*} N^{*}=$ id therefore $k_{i}:=\Pi_{*} K^{i+1}=$ $\Pi_{*}\left(N_{*} N^{*}\right) K^{i+1}=F_{*}\left(N^{*} K^{i+1}\right)$, since $F$ is projective and $\phi$ is étale we can apply the projection formula and obtain

$$
\phi^{*} F_{*}\left(N^{*} K^{i+1}\right)=\widetilde{F}_{*} \xi^{*}\left(N^{*} K^{i+1}\right) .
$$

Furthermore the map $N \circ \xi$ is finite and so

$$
\xi^{*} N^{*} c_{1}\left(\omega_{0}^{\Gamma}\right)^{i+1}=c_{1}\left(\xi^{*} N^{*}\left(\omega_{0}^{\Gamma}\right)\right)^{i+1}=\widetilde{K}^{i+1} .
$$

We conclude by noting that $\phi_{*} \phi^{*}$ is multiplication by $\sigma$.

Proposition 4.3. Following notation of Proposition 2.9 and order elements of $\Delta_{1}$ from 1 to $\delta_{1}$, we have

$$
k_{m}=-\phi_{*} \frac{t_{1}^{m}+\cdots+t_{\delta_{1}}^{m}}{\sigma} .
$$

Proof. From Proposition 4.2, we have reduced the problem to computing $\widetilde{K}$ and then writing pushforward along $\widetilde{F}$. With respect to each component of the stack

$$
\widehat{C}^{\Gamma}=\coprod\left[\mathbb{P}_{\mathbb{C}}^{1} / \operatorname{Aut}\left(C_{0}\right)\right]
$$


we fix coordinates on $\mathbb{P}_{\mathbb{C}}^{1}$ which are compatible with coordinates chosen on $C$. By forgetting the action of $\operatorname{Aut}(\Gamma)$ we keep the same system of coordinates on $\coprod^{\delta+1} \mathbb{P}_{\mathbb{C}}^{1}$ when we consider $\coprod^{\delta+1}\left[\mathbb{P}_{\mathbb{C}}^{1} / H\right]$.

Giving a bundle on a quotient stack $[X / G]$ is equivalent to giving a bundle $U \rightarrow X$ equivariant for the action of $G$. By abuse of notation we still call $\widetilde{\omega}_{0}^{\Gamma}$ any lifting of $\widetilde{\omega}_{0}^{\Gamma}$ on $\coprod^{\delta+1} \mathbb{P}_{\mathbb{C}}^{1}$.

In order to make computations we need to render explicit the action of $H:=E^{\Delta_{1}} \times \mathbb{G}_{\mathbf{m}}^{\Delta_{2}}$ on

$$
\widetilde{F}_{*}\left(\widetilde{\omega}_{0}\right)=H^{0}\left(\coprod^{\delta+1} \mathbb{P}_{\mathbb{C}}^{1}, \widetilde{\omega}_{0}^{\Gamma}\right) .
$$

Set $\Delta:=\Delta_{1} \cup \Delta_{2}$, from the inclusion $\mathbb{G}_{\mathbf{m}} \rightarrow E$ we have a cartesian diagram of stacks

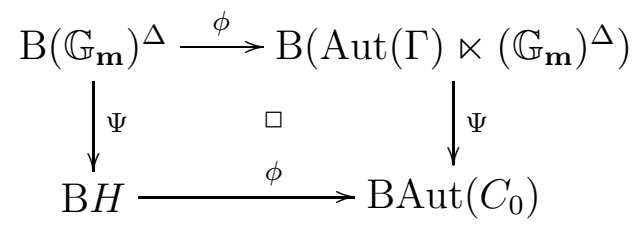

Roughly speaking we can say that we obtain the stacks in the top row by fixing the point 0 on components with a node. The functor $\Psi$ forgets these points. We have the following ring isomorphisms

$$
\begin{aligned}
A_{H}^{*} & \stackrel{\Psi^{*}}{\longrightarrow} A_{\left(\mathbb{G}_{\mathbf{m}}\right)^{\Delta}}^{*} \\
A_{\operatorname{Aut}\left(C_{0}\right)}^{*} & \stackrel{\Psi^{*}}{\longrightarrow} A_{\operatorname{Aut}(\Gamma) \ltimes\left(\mathbb{G}_{\mathbf{m}}\right)^{\Delta} .}^{*}
\end{aligned}
$$

We have defined the classes $t_{\Delta_{1}}, r_{\Delta_{2}}$ in $A_{H}^{*}$. By using the same notation of Section 2, the map $\Psi^{*}$ is the identity on $r_{\Delta_{2}}$. For each vertex $P$ in $\Gamma$ such that $e(P)=1$ the map $\Psi^{*}$ sends $t_{P}$ to $c_{1}^{\mathbb{G}_{\mathbf{m}}}\left(H^{0}\left(\mathbb{P}_{\mathbb{C}}^{1}, \mathcal{O}\left(z_{\infty}\right)\right)\right)$ of the same component, which we still call $t_{P}$. Consequently, with reference to the following diagram

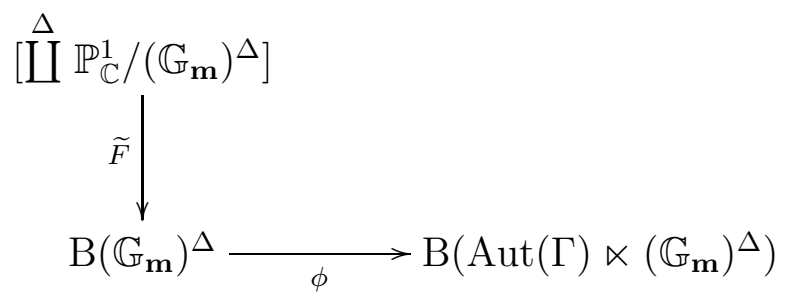


we have reduced the problem to consider the action of $\left(\mathbb{G}_{\mathbf{m}}\right)^{\Delta}$ on

$$
\widetilde{F}_{*}\left(\widetilde{\omega}_{0}^{\Gamma}\right)=H^{0}\left(\coprod \mathbb{P}_{\mathbb{C}}^{1}, \widetilde{\omega}_{0}^{\Gamma}\right)
$$

where, again with abuse of notation, we call $\widetilde{\omega}_{0}^{\Gamma}$ the sheaf $\Psi^{*}\left(\widetilde{\omega}_{0}^{\Gamma}\right)$.

The map $\widetilde{F}$ is the union of maps

$$
\widetilde{F}_{P}:=\left[\mathbb{P}_{\mathbb{C}}^{1} /\left(\mathbb{G}_{\mathbf{m}}\right)^{\Delta}\right] \rightarrow \mathrm{B}\left(\mathbb{G}_{\mathbf{m}}\right)^{\Delta}
$$

where only the component of $\left(\mathbb{G}_{\mathbf{m}}\right)^{\Delta}$ corresponding to $P \in \Delta$ does not acts trivially on $\mathbb{P}_{\mathbb{C}}^{1}$ and the action is

$$
\begin{aligned}
\gamma: \mathbb{G}_{\mathbf{m}} \times \mathbb{P}_{\mathbb{C}}^{1} & \rightarrow \mathbb{P}_{\mathbb{C}}^{1} \\
\left(\lambda,\left[X_{0}, X_{1}\right]\right) & \mapsto\left[X_{0}, \lambda X_{1}\right] .
\end{aligned}
$$

Set

$$
P_{0}:=[0,1], \quad P_{1}:=[1,1], \quad P_{\infty}:=[1,0] .
$$

We have that $\mathcal{F}:=\left(\widetilde{\omega}_{0}\right)^{\vee}$ is the sheaf on $\stackrel{\Delta}{\amalg} \mathbb{P}_{\mathbb{C}}^{1}$ such that restricted:

- to the components with one node it is $\mathcal{F}_{P}:=\left(\omega \otimes \mathcal{O}\left(z_{\infty}\right)\right)^{\vee}$

- to the components with two nodes it is $\mathcal{F}_{P}:=\left(\omega \otimes \mathcal{O}\left(z_{\infty}+z_{0}\right)\right)^{\vee}$

- to the components with three nodes it is $\mathcal{F}_{P}:=\left(\omega \otimes \mathcal{O}\left(z_{\infty}+\right.\right.$ $\left.\left.z_{0}+z_{1}\right)\right)^{\vee}$

where $\omega$ is the canonical bundle on $\mathbb{P}_{\mathbb{C}}^{1}$.

In the following, Chern classes will be equivariant for the action of $\mathbb{G}_{\mathbf{m}}$. For each $P \in \Delta$ let us indicate on each component

$$
\begin{aligned}
K_{P} & :=c_{1}^{\mathbb{G}_{\mathbf{m}}}(\omega) \in A_{\mathbb{G}_{\mathbf{m}}}^{1}\left(\mathbb{P}_{\mathbb{C}}^{1}\right) \\
R_{P} & :=c_{1}^{\mathbb{G}_{\mathbf{m}}}\left(\mathcal{O}\left(z_{\infty}\right)\right) \in A_{\mathbb{G}_{\mathbf{m}}}^{1}\left(\mathbb{P}_{\mathbb{C}}^{1}\right) \\
Q_{P} & :=c_{1}^{\mathbb{G}_{\mathbf{m}}}\left(\mathcal{O}\left(P_{0}\right)\right) \in A_{\mathbb{G}_{\mathbf{m}}}^{1}\left(\mathbb{P}_{\mathbb{C}}^{1}\right)
\end{aligned}
$$

We have

$$
\begin{aligned}
& c_{1}^{\mathbb{G}_{\mathbf{m}}}\left(\mathcal{F}_{\Delta_{1}}\right)=-K_{\Delta_{1}}-R_{\Delta_{1}} \\
& c_{1}^{\mathbb{G}_{\mathbf{m}}}\left(\mathcal{F}_{\Delta_{2}}\right)=-K_{\Delta_{2}}-R_{\Delta_{2}}-Q_{\Delta_{2}} .
\end{aligned}
$$

In order to determine Mumford classes it is then necessary to compute the pushforward classes

$$
\begin{aligned}
& \widetilde{F}_{\Delta_{1} *}\left(-K_{\Delta_{1}}-R_{\Delta_{1}}\right)^{h} \text { and } \\
& \widetilde{F}_{\Delta_{2} *}\left(-K_{\Delta_{2}}-R_{\Delta_{2}}-Q_{\Delta_{2}}\right)^{h}
\end{aligned}
$$

for every natural $h$.

Let us start with computing the push-forward along $\widetilde{F}_{\Delta}$ of every power of $K_{\Delta}=c_{1}^{\mathbb{G}_{\mathbf{m}}}(\omega)$. 
We have

$$
\widetilde{F}_{\Delta_{1} *}\left(\omega^{\vee}\right)=H^{0}\left(\mathbb{P}_{\mathbb{C}}^{1}, \omega^{\vee}\right) .
$$

Now it is necessary to determine the action of $\left(\mathbb{G}_{\mathbf{m}}\right)$ on global sections of $\omega^{\vee}$. Let $z:=X_{1} / X_{0}$ be the local coordinate around $z_{\infty}$, the global sections of $\omega^{\vee}$ are generated as a vectorial space by $\frac{\partial}{\partial z}, z \frac{\partial}{\partial z}, z^{2} \frac{\partial}{\partial z}$. Relatively to this basis, the action of $\mathbb{G}_{\mathbf{m}}$ is given by

$$
\begin{aligned}
\gamma: \mathbb{G}_{\mathbf{m}} \times H^{0}\left(\mathbb{P}_{\mathbb{C}}^{1}, \omega^{\vee}\right) & \rightarrow H^{0}\left(\mathbb{P}_{\mathbb{C}}^{1}, \omega^{\vee}\right) \\
\left(\lambda, a \frac{\partial}{\partial z}+b z \frac{\partial}{\partial z}+c z^{2} \frac{\partial}{\partial z}\right) & \mapsto\left(a \lambda \frac{\partial}{\partial z}+b z \frac{\partial}{\partial z}+c \frac{1}{\lambda} z^{2} \frac{\partial}{\partial z}\right)
\end{aligned}
$$

the multiplicity of the action is therefore $(1,0,-1)$, so the Chern character of $\widetilde{F}_{\Delta *}\left(\omega^{\vee}\right)$ is $e^{t_{\Delta}}+1+e^{-t_{\Delta}}$.

Let us observe that $\omega^{\vee}$ is the tangent bundle relative to $\widetilde{F}_{\Delta}$, so by applying the GRR Theorem we get

$$
1+e^{t_{\Delta}}+e^{-t_{\Delta}}=\widetilde{F}_{\Delta *}\left[\left(e^{-\left(K_{\Delta}\right)}\right)\left(\frac{-K_{\Delta}}{1-e^{K_{\Delta}}}\right)\right]
$$

By applying GRR to the trivial linear bundle we obtain:

$$
1=\widetilde{F}_{\Delta *}\left[\frac{-K_{\Delta}}{1-e^{K_{\Delta}}}\right]
$$

If we subtract the second equation from the first, we obtain

$$
e^{t_{\Delta}}+e^{-t_{\Delta}}=\widetilde{F}_{\Delta *}\left[\left(\frac{1-e^{K_{\Delta}}}{e^{K_{\Delta}}}\right)\left(\frac{-K_{\Delta}}{1-e^{K_{\Delta}}}\right)\right]=\widetilde{F}_{\Delta *}\left[-K_{\Delta} e^{-K_{\Delta}}\right],
$$

from this, by distinguishing between even and odd cases, it follows that

$$
\begin{aligned}
\widetilde{F}_{\Delta *} K_{\Delta}^{2 h} & =0 \\
\widetilde{F}_{\Delta *} K_{\Delta}^{2 h+1} & =-2 t_{\Delta}^{2 h}
\end{aligned}
$$

Let us notice that there exist two equivariant sections of $\widetilde{F}_{\Delta}$ given by the fixed points $z_{0}$ and $z_{\infty}$, that we will call respectively $s_{0}$ and $s_{\infty}$

$$
\mathrm{B}\left(\mathbb{G}_{\mathbf{m}}\right)^{\Delta} \underset{s_{\infty}}{\stackrel{s_{0}}{\longrightarrow}}\left[\mathbb{P}_{\mathbb{C}}^{1} /\left(\mathbb{G}_{\mathbf{m}}\right)^{\Delta}\right]
$$

From the self intersection formula we have

$$
\begin{aligned}
& s_{0}^{*}\left(Q_{\Delta}\right)=-t_{\Delta}, s_{0}^{*}\left(R_{\Delta}\right)=0, s_{0}^{*}\left(K_{\Delta}\right)=t_{\Delta} \\
& s_{\infty}^{*}\left(Q_{\Delta}\right)=0, s_{\infty}^{*}\left(R_{\Delta}\right)=t_{\Delta}, s_{\infty}^{*}\left(K_{\Delta}\right)=-t_{\Delta} ;
\end{aligned}
$$


then by applying the projection formula (see [Fulton p.34) for every cycle $D \in A_{\left(\mathbb{G}_{\mathbf{m}}\right)^{\Delta}}^{*}\left(\mathbb{P}^{1}\right)$ we have

$$
\begin{aligned}
& Q_{\Delta} \cdot D=s_{0 *}(1) \cdot D=s_{0 *}\left(s_{0}^{*} D\right) \\
& R_{\Delta} \cdot D=s_{\infty *}(1) \cdot D=s_{\infty *}\left(s_{\infty}^{*} D\right) .
\end{aligned}
$$

With reference to the previous relations, the Mumford classes are determined on every component (we separate between even and odd cases)

$$
\begin{aligned}
\widetilde{F}_{\Delta *}\left(-K_{\Delta}-R_{\Delta}\right)^{2 h} & =\widetilde{F}_{*}^{\Delta}\left(K_{\Delta}\right)^{2 h}+\sum_{a=1}^{2 h}\left(\begin{array}{c}
2 h \\
a
\end{array}\right) \widetilde{F}_{\Delta *}\left(R_{\Delta}^{a} \cdot K_{\Delta}^{2 h-a}\right) \\
& =\sum_{a=1}^{2 h}\left(\begin{array}{c}
2 h \\
a
\end{array}\right)\left(\widetilde{F}_{\Delta *} s_{\infty *}\right) s_{\infty}^{*}\left(R_{\Delta}^{a-1} \cdot K_{\Delta}^{2 h-a}\right) \\
& =\sum_{a=1}^{2 h}\left(\begin{array}{c}
2 h \\
a
\end{array}\right)(-1)^{a-1} t_{\Delta}^{2 h-1}=-t_{\Delta}^{2 h-1}
\end{aligned}
$$

and in a completely analogous way, we have the following relations

$$
\begin{aligned}
& \widetilde{F}_{\Delta_{1} *}\left(-K_{\Delta_{1}}-R_{\Delta_{1}}\right)^{2 h+1}=t_{i}^{2 h} \\
& \widetilde{F}_{\Delta_{2} *}\left(-K_{\Delta_{2}}-R_{\Delta_{2}}-Q_{\Delta_{2}}\right)^{h}=0 .
\end{aligned}
$$

The last relation allows us to ignore components with two nodes. By following the notation of Proposition 4.2 we notice that

$$
\widetilde{F}_{*} \widetilde{K}^{m+1}=\sum_{P \in \Delta_{1}} \widetilde{F}_{P *}(-1)^{m+1}\left(-K_{P}-R_{P}\right)^{m+1}
$$

and consequently, if we order elements of $\Delta_{1}$ from 1 to $\delta_{1}$, we have

$$
k_{m}=-\phi_{*} \frac{t_{1}^{m}+\cdots+t_{\delta_{1}}^{m}}{\sigma}
$$

Definition of Mumford classes on $\mathfrak{M}_{0}^{\leq 3}$ Now we consider the universal curve

$$
C^{\leq 3} \stackrel{\Pi}{\rightarrow} \mathfrak{M}_{0}^{\leq 3} .
$$

We have seen in Fulg that the pushforward $\Pi_{*}\left(\omega_{0}^{\leq 3}\right)^{\vee}$ is a well defined rank three vector bundle. Consequently from [Kre] Section 3.6 we have that $c_{i}\left(\Pi_{*}\left(\omega_{0}^{\leq 3}\right)^{\vee}\right)=0$ for $i>3$. We fix the following notation

$$
c_{1}:=c_{1}\left(\Pi_{*}\left(\omega_{0}^{\leq 3}\right)^{\vee}\right), \quad c_{2}:=c_{2}\left(\Pi_{*}\left(\omega_{0}^{\leq 3}\right)^{\vee}\right), \quad c_{3}:=c_{3}\left(\Pi_{*}\left(\omega_{0}^{\leq 3}\right)^{\vee}\right) .
$$

We still call $c_{1}, c_{2}, c_{3}$ their restriction to each stratum of $\mathfrak{M}_{0}^{\leq 3}$. 
Definition 4.4. We define in $A^{*}\left(\mathfrak{M}_{0}^{\leq 3}\right)$ Mumford classes $k_{1}, k_{2}, k_{3}$ as follows

$$
k_{1}:=-c_{1}, \quad k_{2}:=2 c_{2}-c_{1}^{2}, \quad k_{3}:=-c_{1}^{3}+3 c_{1} c_{2}-3 c_{3} .
$$

Remark 4.5. From Proposition 2.11, the Chow $\operatorname{ring} A^{*}\left(\mathfrak{M}_{0}^{\leq 3}\right) \otimes \mathbb{Q}$ injects into the product of $A^{*}\left(\mathfrak{M}_{0}^{\Gamma}\right) \otimes \mathbb{Q}$ over trees with at most three edges. Consequently in order to verify that the above is a good definition we only need to prove that the restrictions of Mumford classes to each stratum are the given polynomials.

Proposition 4.6. Let $\Gamma$ be a tree with at most three edges. Set

$$
\begin{aligned}
& c_{1}(\Gamma):=c_{1}\left(\Pi_{*}\left(\omega_{0}^{\Gamma}\right)^{\vee}\right) \\
& c_{2}(\Gamma):=c_{2}\left(\Pi_{*}\left(\omega_{0}^{\Gamma}\right)^{\vee}\right) \\
& c_{3}(\Gamma):=c_{3}\left(\Pi_{*}\left(\omega_{0}^{\Gamma}\right)^{\vee}\right) .
\end{aligned}
$$

and

$$
\begin{aligned}
& n_{1}(\Gamma):=c_{1}(\Gamma), n_{2}(\Gamma):=c_{1}^{2}(\Gamma)-2 c_{2}(\Gamma), \\
& n_{3}(\Gamma):=c_{1}^{3}(\Gamma)-3 c_{1}(\Gamma) c_{2}(\Gamma)+3 c_{3}(\Gamma) .
\end{aligned}
$$

Then we have

$$
k_{1}=-n_{1}(\Gamma) \quad k_{2}=-n_{2}(\Gamma) \quad k_{3}=-n_{3}(\Gamma)
$$

Proof. We consider first of all the case $\mathfrak{M}_{0}^{\Gamma}=\mathfrak{M}_{0}^{0}$. Here we have

$$
\begin{aligned}
& n_{1}(\Gamma)=c_{1}^{\mathbb{P} G l_{2}}\left(\mathfrak{s l}_{2}\right)=0 \\
& n_{2}(\Gamma)=-2 c_{2}^{\mathbb{P} G l_{2}}\left(\mathfrak{s l}_{2}\right) \\
& n_{3}(\Gamma)=0
\end{aligned}
$$

and we can conclude because on $\mathfrak{M}_{0}^{0}$ we have that $k_{1}=k_{3}=0$ and $k_{2}=2 c_{2}^{\mathbb{P} G l_{2}}\left(\mathfrak{s l}_{2}\right)$ (Proposition 4.1).

Now let us consider any other tree $\Gamma$ with at most three edges. With reference to diagram (4), as Chern classes commutes with base changing, we have

$$
\phi^{*} c_{i}\left(\Pi_{*}\left(\omega_{0}^{\Gamma}\right)^{\vee}\right)=c_{i}\left(\widetilde{\Pi}_{*}\left(\widetilde{\omega}_{0}^{\Gamma}\right)^{\vee}\right) .
$$

Given $\Gamma$ we order elements of $\Delta_{1}$ from 1 to $\delta_{1}$. By putting together the above relation and the equation (5), we reduce to show

$$
\operatorname{ch}\left(\widetilde{\Pi}_{*}\left(\widetilde{\omega}_{0}^{\Gamma}\right)^{\vee}\right)=3+\sum_{m=1}^{\infty} \frac{t_{1}^{m}+\cdots+t_{\delta_{1}}^{m}}{m !} .
$$

We recall that the universal curve $\widetilde{C}^{\Gamma}$ on $\widetilde{\mathfrak{M}}_{0}^{\Gamma}=\mathrm{B}\left(E^{\Delta_{1}} \times \mathbb{G}_{\mathbf{m}}^{\Delta_{2}}\right)$ is the quotient stack $\left[C_{0} / E^{\Delta_{1}} \times \mathbb{G}_{\mathbf{m}}^{\Delta_{2}}\right]$. 
Since we have a morphism $\Psi: \mathrm{B}\left(\mathbb{G}_{\mathbf{m}}\right)^{\Delta} \rightarrow \widetilde{\mathfrak{M}}_{0}^{\Gamma}$ such that $\Psi^{*}$ : $A^{*}\left(\widetilde{\mathfrak{M}}_{0}^{\Gamma}\right) \rightarrow A_{\left(\mathbb{G}_{\mathbf{m}}\right)^{\Delta}}^{*}$ is an isomorphism, with reference to the cartesian diagram

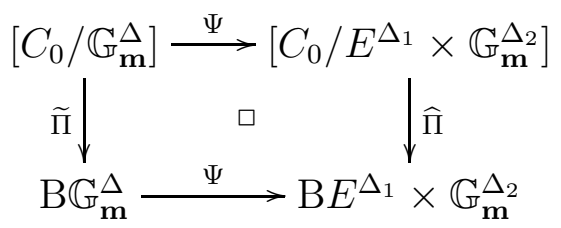

we reduce to consider the pullback sheaf $\Psi^{*}\left(\widetilde{\omega}_{0}^{\Gamma}\right)^{\vee}$ which we still call $\left(\widetilde{\omega}_{0}^{\Gamma}\right)^{\vee}$ as its lifting to $C_{0}$. Standard arguments involving Serre duality, show that $H^{1}\left(C_{0},\left(\widetilde{\omega}_{0}^{\Gamma}\right)^{\vee}\right)=0$, so we have

$$
\operatorname{ch}\left(\widetilde{\Pi}_{*}\left(\widetilde{\omega}_{0}^{\Gamma}\right)^{\vee}\right)=\operatorname{ch}^{\mathbb{G}_{\mathbf{m}}^{\Delta}}\left(H^{0}\left(C_{0},\left(\widetilde{\omega}_{0}^{\Gamma}\right)^{\vee}\right)\right)
$$

On curves of topological type

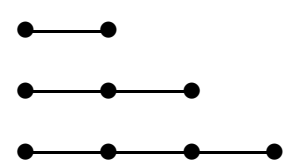

we have that global sections of $\left(\widetilde{\omega}_{0}^{\Gamma}\right)^{\vee}$ are sections of $\mathcal{O}_{\mathbb{P}_{\mathbb{C}}^{1}}(1)$ on extremal components and $\mathcal{O}_{\mathbb{P}_{\mathbb{C}}^{1}}$ on the other components, which agree on nodes. Since $H^{0}\left(\mathbb{P}_{\mathbb{C}}^{1}, \mathcal{O}_{\mathbb{P}_{\mathbb{C}}^{1}}(0)\right)=\mathbb{C}$, global sections of $\left(\widetilde{\omega}_{0}^{\Gamma}\right)^{\vee}$ are sections of $\mathcal{O}_{\mathbb{P}_{\mathbb{C}}^{1}}(1)$ on the two extremal components which are equal on nodes. On each extremal component we fix coordinates $\left[X_{0}^{\prime}, X_{1}^{\prime}\right]$ and $\left[X_{0}^{\prime \prime}, X_{1}^{\prime \prime}\right]$. Sections on $\mathcal{O}_{\mathbb{P}_{\mathbb{C}}^{1}}(1)$ are linear forms

$$
\begin{aligned}
& a_{1} X_{0}^{\prime}+b_{1} X_{1}^{\prime} \\
& a_{2} X_{0}^{\prime \prime}+b_{2} X_{1}^{\prime \prime}
\end{aligned}
$$

which agree at $z_{\infty}=[1,0]$. This happens if and only if $a_{1}=a_{2}$. We have only $\left(\mathbb{G}_{\mathbf{m}}\right)^{\Delta_{1}}$ which does not acts trivially on $H^{0}\left(C_{0},\left(\widetilde{\omega}_{0}^{\Gamma}\right)^{\vee}\right)$ and the action is

$$
\begin{aligned}
\gamma:\left(\mathbb{G}_{\mathbf{m}} \times \mathbb{G}_{\mathbf{m}}\right) \times H^{0}\left(C_{0},\left(\widetilde{\omega}_{0}^{\Gamma}\right)^{\vee}\right) & \rightarrow H^{0}\left(C_{0},\left(\widetilde{\omega}_{0}^{\Gamma}\right)^{\vee}\right) \\
\left(\lambda_{1}, \lambda_{2}\right),\left(a, b_{1}, b_{2}\right) & \mapsto\left(a, \lambda_{1} b_{1}, \lambda_{2} b_{2}\right)
\end{aligned}
$$

so the Chern character of $\widetilde{\Pi}_{*} \omega_{0}^{\vee}$ is

$$
1+e^{t_{1}}+e^{t_{2}}=3+\sum_{m=1}^{\infty} \frac{t_{1}^{m}+t_{2}^{m}}{m !}
$$

as we claimed. 
The last case to consider is when $\Gamma$ equals to

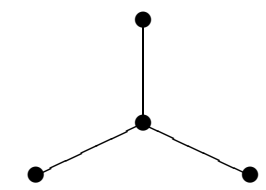

We have that global sections of $\left(\widetilde{\omega}_{0}^{\Gamma}\right)^{\vee}$ are sections of $\mathcal{O}_{\mathbb{P}_{\mathbb{C}}^{1}}(1)$ on extremal components and $\mathcal{O}_{\mathbb{P}_{\mathbb{C}}^{1}}(-1)$ on the central component which agree on nodes. Since $H^{0}\left(\mathbb{P}_{\mathbb{C}}^{1}, \mathcal{O}_{\mathbb{P}_{\mathbb{C}}^{1}}(-1)\right)=0$, global sections of $\left(\widetilde{\omega}_{0}^{\Gamma}\right)^{\vee}$ are sections of $\mathcal{O}_{\mathbb{P}_{\mathbb{C}}^{1}}(1)$ on the three extremal components which are zero on nodes. On each extremal component we fix coordinates $\left[X_{0}^{\prime}, X_{1}^{\prime}\right]$, $\left[X_{0}^{\prime \prime}, X_{1}^{\prime \prime}\right]$ and $\left[X_{0}^{\prime \prime \prime}, X_{1}^{\prime \prime \prime}\right]$. Sections on $\mathcal{O}_{\mathbb{P}_{\mathbb{C}}^{1}}(1)$ are linear forms

$$
a_{1} X_{0}^{\prime}+b_{1} X_{1}^{\prime} \quad a_{2} X_{0}^{\prime \prime}+b_{2} X_{1}^{\prime \prime} \quad a_{3} X_{0}^{\prime \prime \prime}+b_{3} X_{1}^{\prime \prime \prime}
$$

which are zero on nodes if and only if $a_{1}=a_{2}=a_{3}=0$. We have that only $\left(\mathbb{G}_{\mathbf{m}}\right)^{\Delta_{1}}$ does not acts trivially on $H^{0}\left(C_{0},\left(\widetilde{\omega}_{0}^{\Gamma}\right)^{\vee}\right)$ and the action is

$$
\begin{aligned}
\gamma:\left(\mathbb{G}_{\mathbf{m}} \times \mathbb{G}_{\mathbf{m}} \times \mathbb{G}_{\mathbf{m}}\right) \times H^{0}\left(C_{0},\left(\widetilde{\omega}_{0}^{\Gamma}\right)^{\vee}\right) & \rightarrow H^{0}\left(C_{0},\left(\widetilde{\omega}_{0}^{\Gamma}\right)^{\vee}\right) \\
\left(\lambda_{1}, \lambda_{2}, \lambda_{3}\right),\left(b_{1}, b_{2}, b_{3}\right) & \mapsto\left(\lambda_{1} b_{1}, \lambda_{2} b_{2}, \lambda_{3} b_{3}\right)
\end{aligned}
$$

so the Chern character of $\widetilde{\Pi}_{*} \omega_{0}^{\vee}$ is

$$
e^{t_{1}}+e^{t_{2}}+e^{t_{3}}=3+\sum_{m=1}^{\infty} \frac{t_{1}^{m}+t_{2}^{m}+t_{3}^{m}}{m !}
$$

as we claimed.

\section{REFERENCES}

[Fulg] D. Fulghesu,The Stack of Rational Nodal Curves, preprint.

[Fulton] W. Fulton: Intersection theory, Springer-Verlag Berlin, 1998.

[Hrt] R. Hartshorne: Algebraic Geometry, Graduate Text in Mathematics 52, Springer-Verlag New York, Heidelberg, Berlin, 1977.

[Knu] D. Knutson: Algebraic Spaces, Lecture Notes in Math. 203, Springer-Verlag Berlin, 1971.

[Kre] A. Kresch: Cycle groups for Artin stacks; Invent. Math. 138 (1999) 495-536.

[Mum2] D. Mumford: Towards an Enumerative Geometry of the Moduli Space of Curves, in: Arithmetic and geometry, II, Progr. Math. 36 (1983) 271-326.

[Vez] G. Vezzosi: On the Chow ring of the classifying stack of $\mathrm{PGL}_{3, \mathbb{C}} ;$ J. Reine Angew. Math. 523 (2000) 1-54.

[Vis] A. Vistoli: Intersection theory on algebraic stacks and their moduli spaces, Invent. Math. 97 (1989) 613-670.

Department of Mathematics, University of Missouri, Columbia, MO 65211

E-mail address: damiano@math.missouri.edu 